\title{
An Alternative Strategy for the Solution of Heat and Incompressible Fluid Flow Problems via Finite Volume Method
}

\author{
Masoud Nickaeen $^{\mathrm{a}}$, Ali Ashrafizadeh ${ }^{\mathrm{b}}$, Stefan Turek ${ }^{\mathrm{a}}$ \\ ${ }^{a}$ Institute of Applied Mathematics, Dortmund University of Technology, Dortmund, \\ Germany \\ ${ }^{b}$ Faculty of Mechanical Engineering, K.N. Toosi University of Technology, Tehran, Iran
}

\section{running head title: Characteristic-Based Split Finite Volume Method}

Corresponding author: M. Nickaeen, Institute of Applied Mathematics, Dortmund University of Technology, Vogelpothsweg 87, D-44227 Dortmund, Germany. E-mail: masoud.nickaeen@math.tu-dortmund.de 


\section{An Alternative Strategy for the Solution of Heat and Incompressible Fluid Flow Problems via Finite Volume Method}

The characteristic-based split (CBS) method has been widely used in the finite element community to facilitate the numerical solution of Navier-Stokes (NS) equations. However, this computational algorithm has rarely been employed in the finite volume context and the stabilization of the numerical solution procedure has traditionally been addressed differently in volume-based numerical schemes.

In this paper, the CBS-based finite volume algorithm is employed to formulate and solve a number of laminar incompressible flow and convective heat transfer problems. Both explicit and implicit versions of the algorithm are first explained and validated in the context of the solution of a lid-driven cavity problem and a backward facing step (BFS) flow problem. The modified algorithm, capable of modelling the coupling between the momentum and energy balance equations, is then introduced and used to solve a buoyancy-driven cavity flow problem.

Computational results show that the CBS finite volume algorithm can be reliably used in the solution of laminar incompressible heat and fluid flow problems.

Keywords: finite volume, characteristic based split, fractional step, colocated grid, incompressible flow, natural convection 


\begin{tabular}{|c|c|c|c|}
\hline \multicolumn{4}{|c|}{ NOMENCLATURE } \\
\hline$c$ & sound speed & $\beta$ & artificial sound speed \\
\hline$c_{P}$ & specific heat at constant pressure & $\gamma$ & thermal expansion coefficient \\
\hline$E R$ & expansion ratio in BFS flow & $\Delta t$ & time step \\
\hline$g$ & gravitational acceleration & $\Delta x, \Delta y$ & grid spacing in $x$ and $y$ directions \\
\hline$h$ & control volume length scale & $\varepsilon$ & time step control parameter \\
\hline$k$ & fluid thermal conductivity & $\mu$ & fluid viscosity \\
\hline$L$ & characteristic length & $v$ & fluid kinematic viscosity \\
\hline $\mathrm{Nu}$ & Nusselt number & $\rho$ & fluid density \\
\hline$p$ & Pressure & \multirow[b]{2}{*}{ Subscripts } & \\
\hline $\operatorname{Pr}$ & Prandtl number $(=v / \alpha)$ & & \\
\hline$R a$ & Rayleigh number $\left(=g \gamma \Delta T L^{3} / v \alpha\right)$ & $P, N, E, W, S$ & nodal point values \\
\hline $\operatorname{Re}$ & Reynolds number & n, e, w, s & integration point values \\
\hline$t$ & Time & $1,2, \ldots$ & corner nodes \\
\hline$T$ & temperature & conv & convection related value \\
\hline$u$ & $x$-velocity component & diff & diffusion related value \\
\hline$U$ & mass flux $(=\rho u)$ & h, c & hot and cold walls \\
\hline$v$ & $y$-velocity component & $\infty$ & far field value \\
\hline$V$ & volume & \multirow[b]{2}{*}{ Superscripts } & \\
\hline$x$ & horizontal coordinate & & \\
\hline$y$ & vertical coordinate & $\mathrm{n}, \mathrm{n}+1$ & old and new iterations \\
\hline$X_{1}, X_{2}, X_{3}$ & characteristic lengths in BFS flow & $*$ & intermediate field value \\
\hline$\alpha$ & thermal diffusivity & , & non-dimensional value \\
\hline
\end{tabular}




\section{INTRODUCTION}

Over the last decade, Computational Fluid Dynamic (CFD) simulation of the NS equations has become a basic tool for industrial design and analysis. Computational Fluid Dynamic analysis is now common in a diverse range of applications such as aerospace engineering and power generation, chemical and material engineering processes, and automotive industry just to name a few. An example of the application of CFD in the real world engineering problems is presented in [1], in which the automobile paint curing process simulation is discussed.

The wide spread use of CFD in the solution of science and engineering problems is partly due to the development of robust and accurate discretization schemes in the context of finite volume method [2]. A common symptom in the numerical solution of incompressible NS equations is the appearance of unrealistic oscillations in convection dominated flows. It is well-known that the central difference-based discretization in FVM and standard finite element Galerkin method fail to properly model the advection terms in the fluid flow governing equations.

The oscillatory numerical solutions can be prevented by employing the upwinding strategies originally developed in the finite difference context $[3,4]$. In the finite element framework, Petrov-Galerkin and Galerkin least square schemes [5] are solutions with similarities to those used in the finite volume community. Numerical schemes such as characteristic Galerkin or Taylor-Galerkin [5] are other remedies for the problem of advection numerical modelling.

In the present study the concept of characteristic-Galerkin procedure is employed to stabilize the solution of convection diffusion equations. We perform the temporal 
discretization of the modified momentum equations using the characteristic concept. This leads to the introduction of additional second order diffusion-like terms that prevent the numerical oscillations normally occurred with central approximation of convection terms in convection dominated flows. Therefore, we take advantage of second order approximations for both convection and diffusion modelling without using any additional upwinding methods to control the numerical oscillations.

The necessity of proper pressure-velocity coupling in incompressible flows (pressure stabilization), has been another major difficulty in the numerical solution of incompressible NS equations. Proper remedies and algorithms in this regard have been devised in both finite volume [2] and finite element [5] contexts. One particular family of techniques, also used in this study, is the class of splitting methods. The splitting process was initially introduced by Chorin [6] for incompressible flow problems in the finite difference framework. Afterwards, the splitting method was employed in the finite element context and used for the solution of different incompressible flow problems [7-9]. Characteristic Based Split (CBS) is a variant of splitting methods which was first introduced in 1995 by Zienkiewicz and Codina [10] to solve the fluid flow problems. The foremost advantage of this method is the capability of solving either incompressible or compressible subsonic and supersonic flows by the same algorithm [5]. Further developments and applications of the CBS finite element method can be found in the review papers by Zienkiewicz et al. [11] and Nithiarasu et al. [12] and recently by Nithiarasu [13]. This method has been also developed in the mesh free context for the solution of incompressible NS equations [14].

The CBS has been recently extended and employed in the finite volume context for the solution of incompressible NS equations. In the semi-implicit version of the CBS, 
introduced in [15], a Poisson pressure equation needs to be solved for the unknown pressure field. In the fully explicit version of the scheme, introduced in [16], artificial compressibility approach is employed. Conceptually, the CBS finite volume scheme can be considered as a variant of the well established family of fractional step methods. The idea is to obtain the solution of the NS equations through the solutions of a number of easier equations. The numerical algorithm is often implemented in three steps; i.e. the calculation of an intermediate velocity field, the calculation of the pressure field corresponding to a divergence-free velocity field and, finally, the projection of the intermediate velocity on a divergence-free velocity field. A comparison between the semi-implicit and fully explicit variants of the CBS finite volume method, which defer only in the pressure update step, is presented in [17].

In this paper the above-mentioned variants of the CBS finite volume method are reviewed and then employed to not only simulate the coupling between the mass and momentum equations, but also the coupling between the momentum and energy balances in a free convection heat transfer problem.

The paper is organized as follows: the next section, section 2, presents the governing equations and a description of the CBS finite volume method. The differences between the two versions of the CBS scheme are also presented in this section. In the next section, performance of the CBS finite volume method is investigated through the solution of three steady incompressible flow problems, i.e., lid-driven cavity flow, backward facing step flow, and buoyancy-driven flow. Finally, some conclusions are drawn in the last section. 


\section{NUMERICAL SOLUTION OF THE NS EQUATIONS}

\subsection{Governing Equations}

The governing equations for the viscous incompressible flow are expressions for the conservation of mass, momentum components and energy as follows:

Mass conservation

$$
\frac{\partial \rho}{\partial t}+\frac{\partial\left(U_{i}\right)}{\partial x_{i}}=0
$$

Momentum conservation

$$
\frac{\partial\left(U_{i}\right)}{\partial t}+\frac{\partial\left(U_{i} u_{j}\right)}{\partial x_{j}}=-\frac{\partial p}{\partial x_{i}}+\frac{\partial}{\partial x_{j}}\left(\mu \frac{\partial u_{i}}{\partial x_{j}}\right)+\rho g_{i}
$$

Energy conservation

$$
\frac{\partial\left(\rho c_{p} T\right)}{\partial t}=-\frac{\partial\left(\rho u_{i} c_{p} T\right)}{\partial x_{i}}+\frac{\partial}{\partial x_{i}}\left(k \frac{\partial T}{\partial x_{i}}\right)
$$

In the above equations $U_{i}=\rho u_{i}$ is the $i^{\text {th }}$ mass flux component. It should be noted that the transient density term in the continuity equation, Eq. 1, can be replaced by the following relation under isentropic flow assumption

$$
\frac{\partial \rho}{\partial t}=\frac{1}{c^{2}} \frac{\partial p}{\partial t}
$$

In this Equation c is the wave (sound) speed.

\subsection{The CBS Method}

The CBS is developed here for the solution of incompressible NS equations in the finite volume context. 


\subsection{Temporal Discretization along Characteristics}

The first aspect of the CBS scheme is the discretization along the characteristics [5]. In particular, the temporal discretization of the momentum equation, Eq. 2, along the characteristics, using a backward Euler discretization method, results in the following time discrete equation [5]:

$$
\begin{aligned}
U_{i}^{n+1}-U_{i}^{n}=-\Delta t\left\{\frac{\partial\left(U_{i} u_{j}\right)}{\partial x_{j}}-\frac{\partial}{\partial x_{j}}\left(\mu \frac{\partial u_{i}}{\partial x_{j}}\right)+\frac{\partial p}{\partial x_{i}}-\rho g_{i}\right\}^{n} & \\
+ & \frac{\Delta t^{2}}{2} u_{k} \frac{\partial}{\partial x_{k}}\left\{\frac{\partial\left(U_{i} u_{j}\right)}{\partial x_{j}}-\frac{\partial}{\partial x_{j}}\left(\mu \frac{\partial u_{i}}{\partial x_{j}}\right)+\frac{\partial p}{\partial x_{i}}-\rho g_{i}\right\}^{n}
\end{aligned}
$$

Note that additional diffusion-like terms are introduced in the momentum equations. These terms are required to stabilize the velocity solution in convection-dominated flows, and hence in the discretization step, central difference scheme can be used to discretize the mass fluxes and the velocity gradients. Pressure stabilization is obtained via fractional step method, through the solution of an approximate momentum equation which may be obtained without considering the pressure term. Equation (5) can be modified as follows:

$$
\begin{aligned}
U_{i}^{*}-U_{i}^{n}=-\Delta t\left\{\frac{\partial\left(U_{i} u_{j}\right)}{\partial x_{j}}\right. & \left.-\frac{\partial}{\partial x_{j}}\left(\mu \frac{\partial u_{i}}{\partial x_{j}}\right)-\rho g_{i}\right\}^{n} \\
& +\frac{\Delta t^{2}}{2} u_{k} \frac{\partial}{\partial x_{k}}\left\{\frac{\partial\left(U_{i} u_{j}\right)}{\partial x_{j}}-\frac{\partial}{\partial x_{j}}\left(\mu \frac{\partial u_{i}}{\partial x_{j}}\right)-\rho g_{i}\right\}^{n}
\end{aligned}
$$

In the absence of pressure gradient, the velocity field $U^{*}$ from Eq. (6) does not satisfy the divergence free constraint of the incompressible flows. This is called intermediate velocity and the solution to Eq. (6) is the first step of the fractional step algorithm. It should be noted that Eq. (6) will be solved by an explicit time step applied to the fully discretized 
form.

Reformulating the momentum conservation Eq. (2) using intermediate velocities in Eq. (6), one can obtain the following equation for the final velocities:

$$
U_{i}^{n+1}-U_{i}^{*}=-\Delta t \frac{\partial p^{n}}{\partial x_{i}}+\frac{\Delta t^{2}}{2} u_{k} \frac{\partial}{\partial x_{k}}\left(\frac{\partial p^{n}}{\partial x_{i}}\right)
$$

Since the pressure is still unknown, this equation can be used after the solution of an appropriate pressure equation to obtain final velocities. In fact, solution of Eq. (7) is the third step of the proposed fractional step algorithm.

To enforce the divergence free constraint and to find the pressure, the divergence operator is used to operate on Eq. (7):

$$
\frac{\partial}{\partial x_{i}}\left\{U_{i}^{n+1}-U_{i}^{*}=-\Delta t \frac{\partial p^{n}}{\partial x_{i}}+\frac{\Delta t^{2}}{2} u_{k} \frac{\partial}{\partial x_{k}}\left(\frac{\partial p^{n}}{\partial x_{i}}\right)\right\}
$$

At this point, two versions of the CBS method studied in this work can be obtained from the above equation. In order to enforce the incompressibility constraint at each time step, the unknown velocity divergence, i.e. $\frac{\partial U_{i}^{n+1}}{\partial x_{i}}$, is assumed to be zero for incompressible flows, and the final equation for pressure update reads:

$$
\frac{\partial}{\partial x_{i}}\left(\frac{\partial p}{\partial x_{i}}\right)^{n+1}=\frac{1}{\Delta t} \frac{\partial U_{i}^{*}}{\partial x_{i}}
$$

Equation (9) is a Poisson pressure equation which can be solved implicitly for the unknown pressure. The implicit solution of Eq. (9) combined with the explicit solution of Eq. (6) leads to the semi-implicit version of the proposed algorithm [15]. 
To devise the fully explicit version of the CBS method [16], the unknown velocity divergence term in Eq. (8) is obtained from a continuity-like equation. The transient density term is retained in the mass conservation Eq. (1), and is related to the time variation of pressure through the introduction of an artificial sound speed $\beta$, which has a finite value, as follows

$$
\frac{\partial \rho}{\partial t}=\frac{1}{\beta^{2}} \frac{\partial p}{\partial t}
$$

Thus the fully explicit version of the algorithm employs the following explicit formula for the pressure update:

$$
\frac{1}{\beta^{2}}\left(p^{n+1}-p^{n}\right)=\Delta t\left[-\frac{\partial U_{i}^{*}}{\partial x_{i}}+\Delta t \frac{\partial}{\partial x_{i}}\left(\frac{\partial p}{\partial x_{i}}\right)^{n}\right]
$$

This equation has no physical meaning at each time step, but results in the satisfaction of the incompressibility constraint at steady state. The solution of any of the pressure equations (9) or (11) is done in the second step of the fractional step method.

The energy equation, as a convection diffusion equation for the temperature unknown, is also discretized temporally along the characteristics [5]

$$
\begin{aligned}
\rho c_{P}\left(T^{n+1}-T^{n}\right)=-\Delta t & \left\{\frac{\partial\left(\rho u_{i} c_{P} T\right)}{\partial x_{i}}-\frac{\partial}{\partial x_{i}}\left(k \frac{\partial T}{\partial x_{i}}\right)\right\}^{n} \\
& +\frac{\Delta t^{2}}{2} u_{k} \frac{\partial}{\partial x_{k}}\left\{\frac{\partial\left(\rho u_{i} c_{P} T\right)}{\partial x_{i}}-\frac{\partial}{\partial x_{i}}\left(k \frac{\partial T}{\partial x_{i}}\right)\right\}^{n}
\end{aligned}
$$

The solution of energy equation can be considered as the fourth step of our fractional step CBS scheme in non-isothermal flows. In this paper, however, the natural convection problem is simulated through the use of Boussinesq approximation and therefore, temperature and velocity fields are coupled. To maintain proper velocity-temperature 
coupling, the energy equation is solved after each velocity update during the CBS iterations.

The above equations, Eqs. (6)-(12), represent the basic steps of the CBS algorithm. Finite volume method is then employed to discretize the space derivatives on a colocated structured grid arrangement. It is important to note that central difference scheme has been used for all the velocity and pressure approximations. This is allowed here because the stabilization required for the convective terms is done via introduction of some additional diffusion terms by using the characteristics method.

\subsection{Spatial Discretization Using FVM}

\section{Colocated Grid}

To start the finite volume spatial discretization, the solution domain must be divided into control volumes. In this study, colocated, structured grids are used in a Cartesian coordinate system. The computational grid is generated based on the cell-centered scheme and employs common finite volume notations. An example for a grid generated in a rectangular domain is shown in Figure 1.

In Figure 1, capital and small letters represent the control volume centers and the Integration Points (IP), respectively. Numbers from 1 to 4 are the four corners of a typical control volume.

\section{Finite Volume Discretization: Step 1}

To carry out the first discretization step of the CBS scheme, we integrate Eq. (6) over a control volume $V p$ associated with cell $\mathrm{p}$ shown in Figure 1. Using the Divergence 
theorem, the volume integrals change to surface integrals and this introduces the IP quantities into the equation. For example, the spatial semi-discrete form of the convection terms in the $\mathrm{x}$-momentum equation ( $\mathrm{i}=1$ in Eq. (6)) is written as follows:

$$
\begin{aligned}
\int_{V_{p}} \frac{\partial\left(U_{i} u_{j}\right)^{n}}{\partial x_{j}} d V_{p}=\int_{V_{p}}[ & \left.\frac{\partial(U u)^{n}}{\partial x}+\frac{\partial(U v)^{n}}{\partial y}\right] d V_{p}= \\
& \Delta y_{p}\left[(U u)_{e}-(U u)_{w}\right]^{n}+\Delta x_{p}\left[(U v)_{n}-(U v)_{s}\right]^{n}
\end{aligned}
$$

The IP velocities are related to nodal values via linear approximation. For example, the velocity on the right face of the cell p in Figure 1 is approximated as follows:

$$
u_{e}=\frac{\frac{u_{E}}{\Delta x_{P}}+\frac{u_{P}}{\Delta x_{E}}}{\frac{1}{\Delta x_{P}}+\frac{1}{\Delta x_{E}}} .
$$

In Eq. (14), $\Delta x$ and $\Delta y$ represent the cell-face areas in Figure 1. All other IP values are treated similarly with simple geometrical interpolations.

To complete the first step of the discretization procedure, we have to approximate the diffusion fluxes. These fluxes are also approximated using the central difference scheme. Discretization of additional diffusion terms in Eq. (6) requires the values of the velocities at the control volume corners. These corner values are approximated using the weighted average of the surrounding nodal values. For example, the x-velocity of point 1 in Figure 1 is approximated as follows:

$$
u_{1}=\frac{\left(\frac{u_{N}}{V_{N}}+\frac{u_{N E}}{V_{N E}}+\frac{u_{E}}{V_{E}}+\frac{u_{P}}{V_{P}}\right)}{\left(\frac{1}{V_{N}}+\frac{1}{V_{N E}}+\frac{1}{V_{E}}+\frac{1}{V_{P}}\right)}
$$


As it is mentioned earlier, we use central differences to approximate both convective and diffusive fluxes. Therefore, the discretization procedure yields an explicit equation relating the cell-centered value of intermediate velocities to that of the cellcentered velocities and their neighbours (E, W, N, S, NE, NW, SE and SW in Figure 1) from the previous time step.

\section{Finite Volume Discretization: Step 2}

In the second step of the CBS method, the pressure is obtained from either Eq. (9) or (11) for semi-implicit and fully explicit versions, respectively. The finite volume discretization of these equations is straightforward. Again, after integrating over control volumes and introducing the surface integrals into the equation, the central difference scheme is used to discretize pressure gradients on the IPs.

\section{Finite Volume Discretization: Step 3}

From steps 1 and 2 we have the intermediate velocities and the pressure, respectively. Using these values, one can proceed with the finite volume discretization of Eq. (7). Integrating Eq. (7) over the control volume, the following equation can be obtained:

$$
\int_{V_{p}}\left(U_{i}^{n+1}-U_{i}^{*}\right) d V_{p}=\int_{V_{p}}\left[-\Delta t \frac{\partial p^{n}}{\partial x_{i}}+\frac{\Delta t^{2}}{2} u_{k} \frac{\partial}{\partial x_{k}}\left(\frac{\partial p^{n}}{\partial x_{i}}\right)\right] d V_{p}
$$

An explicit solution of this equation is carried out to obtain the final velocities.

\section{Finite Volume Discretization: Step 4}

Finite volume discretization of Eq. (12) is the same as what we have done for intermediate velocities. Here, the IP temperature values and temperature gradients are approximated 
using the central difference scheme. Using an approximation like Eq. (15), the required temperature values at control volume corners are also obtained.

\subsection{Boundary Conditions}

In the present study, the velocity boundary conditions are applied in the first step of the algorithm. Thus, the real velocity boundary conditions have been used to obtain the intermediate velocities of the first step.

The solution of the second step requires the implementation of appropriate pressure boundary conditions. In the case of known pressure values on the boundary, outflow boundary condition in a channel flow, the implementation is simple and straightforward. In other cases with velocities or their gradients as known boundary conditions, the proper boundary condition for the pressure equation is applied using velocity components normal to the physical boundary of interest. Since the cell-centered grid is employed, the required pressure values on the control surfaces are estimated using linear extrapolation from the inner neighbouring nodes.

In the third step, the extrapolated IP pressure values of the second step are again used to obtain the correct velocities.

The application of different boundary conditions to the energy equation is also simple on the structured finite volume grids and can be done in the fourth step of the CBS scheme.

\subsection{Time Step Calculation}

Iterative solution of the explicit modified momentum equations of the first step, Eq. (6), is subjected to time step limitations and so is the overall CBS scheme. The permissible time 
step is obtained based on the time scales of the convection and diffusion terms $[15,16]$. The critical time step is calculated as follows:

$$
\Delta t_{c r i t}=\min \left(\Delta t_{\text {conv }}, \Delta t_{\text {diff }}\right)
$$

In Eq. (17), $\Delta t_{\text {conv }}$ and $\Delta t_{\text {diff }}$ are the convection and diffusion time steps respectively, and are calculated as follows:

Semi-implicit method

$$
\Delta t_{\text {conv }}=\frac{h}{u_{\text {conv }}}, \Delta t_{\text {diff }}=\frac{h}{u_{\text {diff }}}
$$

Fully explicit method

$$
\Delta t_{\text {conv }}=\frac{h}{u_{\text {conv }}+\beta}, \Delta t_{\text {diff }}=\frac{h}{u_{\text {diff }}}
$$

In Eq. (19), $\beta=\max \left(\varepsilon, u_{\text {conv }}, u_{\text {diff }}\right)$ is the artificial sound speed and $\varepsilon$ is a constant (taken as 0.5 here), $u_{\text {conv }}$ and $u_{\text {diff }}$ are the convection and diffusion velocities, respectively. For isothermal flows, these velocities are calculated as follows:

$$
u_{\text {conv }}=\sqrt{u_{i} u_{i}}, u_{\text {diff }}=\frac{2 v}{h}
$$

And, for non-isothermal flows as

$$
u_{\text {conv }}=\sqrt{u_{i} u_{i}}, u_{d i f f}=\min \left(\frac{2 v}{h}, \frac{2 \alpha}{h}\right)
$$

In the above equations, $h$ is a control volume length scale. In a two dimensional uniform grid, $h$ is simply the width of the control volume. On non-uniform grids; however, different length scales can be used. Further information regarding the definition of artificial sound speed can be found in [16]. 
To ensure the stability of the method, the final global time step is chosen as a fraction of $\Delta t_{c r i t}$. In other words, a safety factor varying between 0 and 1 is imposed on the calculated time step in Eq. (17).

\subsection{CBS Iterative Algorithm}

The proposed CBS finite volume method can be summarized as follows

(1) Initialize $u_{i}^{n}, p^{n}$ and $T^{n}$ and calculate $\Delta t_{\text {crit }}$ from Eq. (17)

(2) Solve Eq. (6) to obtain the intermediate velocities $u_{i}^{*}$

(3) Solve either Eq. (9) or Eq. (11) to obtain the new pressure $p^{n+1}$

(4) Use the intermediate velocities from step (2) and the pressure field from step (3) to calculate the new velocities $u_{i}^{n+1}$ from Eq. (7)

(5) Solve Eq. (12) to obtain the new temperature $T^{n+1}$

(6) Check for convergence of $u_{i}^{n+1}, p^{n+1}$ and $T^{n+1}$. If solution is converged terminate the CBS loop, otherwise update $\Delta t_{\text {crit }}$ from Eq. 17 and continue to step (2)

It should be noted that for the problems with independent velocity and temperature fields, step (5) can be also done outside the main CBS iteration loop after the converged velocities are determined.

\section{RESULTS AND DISCUSSION}

In this section, some benchmarking has been done for incompressible laminar flow problems to investigate the performance of the proposed fully explicit and semi-implicit versions of the CBS scheme. In the first test case, the lid-driven cavity flow has been considered. Numerical solutions at different Reynolds numbers are presented and compared 
with available literature. In the second example, steady state Backward Facing Step (BFS) flow has been simulated at different Reynolds numbers. Reattachment and recirculation lengths are taken as a measure of validating accuracy of the methods in this problem. Finally, buoyancy-driven cavity flow is solved using the CBS finite volume method. This is a standard natural convection problem in which, Boussinesq approximation is employed to take into account buoyancy forces due to the density differences in the flow.

In order to continue with the results, dimensionless forms of the governing equations are employed. In the first two test cases, the gravitational forces are neglected with respect to other terms of the momentum equation and the following non-dimensional parameters are used:

$$
\begin{aligned}
& x_{i}^{\prime}=\frac{x_{i}}{L}, u_{i}^{\prime}=\frac{u_{i}}{u_{\infty}}, t^{\prime}=\frac{t u_{\infty}}{L}, p^{\prime}=\frac{p}{\rho_{\infty} u_{\infty}^{2}} \\
& \rho^{\prime}=\frac{\rho}{\rho_{\infty}}, c^{\prime 2}=\frac{c^{2}}{u_{\infty}^{2}}
\end{aligned}
$$

And the Reynolds number is defined as

$$
\operatorname{Re}=\frac{\rho_{\infty} u_{\infty} L}{\mu_{\infty}} .
$$

For natural convection problem, in which gravitational forces are important, the following non-dimensional parameters are used:

$$
\begin{aligned}
& u_{i}^{\prime}=\frac{u_{i} L}{\alpha}, t^{\prime}=\frac{t \alpha}{L^{2}}, p^{\prime}=\frac{p L^{2}}{\rho_{\infty} \alpha^{2}}, T^{\prime}=\frac{T-T_{C}}{T_{H}-T_{C}} \\
& \mu^{\prime}=\frac{\mu}{\mu_{\infty}}, k^{\prime}=\frac{k}{k_{\infty}}, c_{P}^{\prime}=\frac{c_{P}}{c_{P \infty}}
\end{aligned}
$$

And the non-dimensional numbers of interest are Rayleigh and Prandtl:

$$
R a=\frac{g \gamma \Delta T L^{3}}{v \alpha}, \operatorname{Pr}=\frac{v}{\alpha}
$$

For further details regarding the non-dimensional parameters one should refer to [18]. 


\subsection{Test Case I (Lid-Driven Cavity)}

Steady state lid-driven cavity flow is considered here as the first test case. This two dimensional square cavity is shown in Figure 2a. This problem is solved using two different non-uniform structured meshes as shown in Figures 2b and 2c. These computational grids which consist of $85 \times 85$ (Mesh 1) and $129 \times 129$ (Mesh 2) control volumes are clustered near the corners of the solution domain.

Mesh 1 is used to solve the cavity flow at Reynolds numbers 400 and 1000. In Figures 3 and 4, the velocity distributions at various Reynolds numbers are compared with the benchmark solution of Ghia et al. [19]. The vertical velocity (v) components along the mid-horizontal line are compared in Figure 3 and the horizontal velocity $(u)$ components along the mid-vertical line are compared in Figure 4. The results of both CBS finite volume methods are in close agreement with the benchmark solution.

To obtain accurate solutions at Reynolds number 5000, the finer mesh, mesh 2, is used. Again in this case the horizontal and vertical mid-plane velocities are compared with those of Ghia et al. [19] in Figure 5.

In Table 1, the values of maximum and minimum velocities and their corresponding positions along the mid lines in the cavity are also compared with [19]. These results are obtained from the fully explicit version of the CBS finite volume method. Table 1 illustrates the appropriate convergence behaviour of the method with respect to grid refinement and also provides the results for validation of our proposed method.

\subsection{Test Case II (Backward Facing Step)}

In the second test case, solution of Backward Facing Step (BFS) flow, shown in Figure 6, is investigated. It is a $2 \mathrm{D}$ channel flow with the inlet width $\mathrm{h}$ and the outlet width $\mathrm{H}$. The 
length of the flow passage is equal to $26 \mathrm{H}$. Depending on the value of the flow Reynolds number, different recirculation zones occur in the flow as shown schematically in Figure 6.

In this problem, a fully-developed parabolic u-velocity profile is prescribed at the inlet of the channel and zero-normal velocity derivatives are imposed at the exit. The location of the outflow boundary is chosen to be sufficiently far downstream of the step so that it does not affect the position of the recirculation zones. All other walls are subjected to the no-slip boundary condition.

Expansion ratio $E R=H / h$ is set to 1.94 to be able to compare the computational results of CBS finite volume method with the experimental results of Armaly et al. [20].

A non-uniform structured mesh, Figure 7, is employed here to investigate the reattachment and separation lengths for Reynolds numbers from 100 to 800 . Reynolds number for the flow configuration in Figure 6 is defined as follows

$$
\operatorname{Re}=\frac{\rho_{\infty} U_{\infty} 2 h}{\mu_{\infty}} .
$$

Grid independent results, non-dimensional separation and reattachment lengths, of the CBS method are compared with the experimental data of Armaly et al. [20] in Figure 8. Since the computational results of both CBS methods are very similar, here we have only shown the results from the fully explicit version. The results of the fully explicit CBS method are in close agreement with the experimental data up to Reynolds 500. For flows with Reynolds number above 500, the reattachment and the separation lengths $X_{1}$ and $X_{2}$ deviate from the experimental results. These deviations for the Reynolds number above 500 are probably due to the three-dimensional effects that are neglected in the present 2D analysis. 
The computational cost (time) and number of iterations for different Reynolds numbers are presented in Table 2 for both CBS procedures. To ensure that the grid independent solution is obtained, mesh refinement is done for each Reynolds number. The non-dimensional reattachment length is then calculated and compared with the experimental data of Armaly et al. [20]. The results of Table 2 show that both methods provide accurate solutions. The semi-implicit solver is faster than the fully explicit one with respect to computational time and the number of iterations required to reach the same desired accuracy in both schemes is larger in the fully explicit version.

\subsection{Test Case III (Buoyancy-Driven Cavity)}

Solution of buoyancy-driven cavity flow is presented here for the first time using CBS finite volume method. The fluid fills a square cavity with the vertical walls being kept at different temperatures, and two horizontal adiabatic walls as shown in Figure 9. Fluid is assumed to be viscous, incompressible and Boussinesq-approximated. No-slip velocity boundary conditions are applied on all walls. A local temperature difference creates a local density difference within the fluid and results in fluid motion because of the buoyancy forces. These forces have to be included in the momentum equation, and the nondimensional parameter in this case becomes the Rayleigh number:

$$
R a=\frac{g \gamma\left(T_{h}-T_{c}\right) L^{3}}{v \alpha}
$$

In Eq. (27), $g$ is the magnitude of the gravitational acceleration, $\gamma$ the coefficient of thermal expansion and, $T_{h}=1, T_{c}=0$ are the hot and cold wall temperatures, respectively. The Prandtl number is also used as it is defined in Eq. (25). 
The series of meshes used for this problem have the same non-uniform structure as those in the lid-driven cavity flow (Figure 2). The fluid in the cavity is air and the problem is solved for four different Rayleigh numbers, i.e., $10^{3}, 10^{4}, 10^{5}$ and $10^{6}$.

Figure 10 illustrates a set of plots for each Rayleigh number. These plots are streamlines, $u$ and $v$ velocity contour and temperature contours. Since the results of both fully explicit and semi-implicit algorithms are quite similar, the results are presented in Figure 10 only once for each $R a$ number.

In the next step, grid independent behaviour of the CBS finite volume methods for the solution of buoyancy-driven flow is studied. A series of four non-uniform structured grids, $(21 \times 21,41 \times 41,81 \times 81,161 \times 161)$, have been employed at $R a=10^{3}$ and the maximum values (max) of vertical and horizontal velocities along the mid-height and midwidth lines are summarized in Table 3, respectively. Both semi-implicit and fully explicit algorithms perform well and even a grid size of $21 \times 21$ can produce reasonable results which compare well with those of other refined grids.

In Table 4, the fully explicit version is used to compare a number of important quantities for buoyancy-driven flow with the numerical results of [21] and [22]. These quantities are the maximum horizontal velocity and its associated $y$ along the mid-width, the maximum vertical velocity and its associated $x$ along the mid-height and maximum (Max.) and minimum (Min.) Nusselt numbers $(\mathrm{Nu})$ along the hot wall. A $81 \times 81$ grid is used for Rayleigh numbers up to $10^{5}$ and a $161 \times 161$ grid for $R a=10^{6}$. Comparison of the CBS results with the benchmark results shows a close agreement for $10^{3}<R a<10^{5}$. For $R a=10^{6}$ however, the CBS method under predicts the maximum and minimum values 
of the flow quantities, while still maintains the right prediction for the position of these quantities.

\section{CONCLUSION}

A finite volume method based on the characteristic based split approach, commonly employed in finite element communities, is introduced in this paper. The proposed method employs characteristic based stabilization for numerical treatment of the convection terms in momentum and energy equations. Consequently, all integration point values are approximated with simple geometrical interpolation formulas without using any upwinding methods for convection modelling. The pressure stabilization in the proposed method is achieved via a fractional step method. The algorithm is presented in two different fully explicit and semi-implicit forms which differ only in the pressure update step. The proposed CBS finite volume method is then used to solve laminar incompressible fluid flow and heat transfer problems on structured colocated grids. Lid-driven cavity, backward facing step and the buoyancy-driven cavity flows are successfully simulated in the laminar regime to validate the proposed algorithm. Comparison with the benchmark numerical and experimental results shows that the predictions in all test cases are satisfactory. 


\section{REFERENCES}

1. A. Ashrafizadeh, R. Mehdipour, and M. Rezvani, An Efficient and Accurate Numerical Simulation Method for the Paint Curing Process in Auto Industries, Proc. International Conference on Applications and Design in Mechanical Engineering (ICADME), Penang, Malaysia, 2009.

2. S. Acharya, B. R. Baliga, K. Karki, J. Y. Murthy, C. Prakash, and S. P. Vanka, PressureBased Finite-Volume Methods in Computational Fluid Dynamics, Journal of heat transfer, vol. 129, pp. 407-424, 2007.

3. E. Isaacson, R. Courant, and M. Rees, On the Solution of Non-linear Hyperbolic Differential Equations by Finite Differences, Comm. Pure Appl. Math., vol. 5, pp. 243-255, 1952.

4. D.B. Spalding, A Novel Finite Difference Formulation for Differential Equations Involving both First and Second Derivatives, Int. J. Numer. Meth. Engrg., vol. 4, pp. 551-559, 1972.

5. O. C. Zienkiewicz, R. L. Taylor, and P. Nithiarasu, The Finite Element Method for Fluid Dynamics, $6^{\text {th }}$ ed., Butterworth and Heinemann, Oxford, 2005.

6. A.J. Chorin, Numerical solution of Navier-Stokes Equations, Mathematics of Computation, vol. 22, pp. 745-762, 1968.

7. G.E. Schneider, G.D. Raithby, and M.M. Yovanovich, Finite Element Analysis of Incompressible Flow Incorporating Equal Order Pressure and Velocity Interpolation, In C. Taylor, K. Morgan and C.A. rebbia, editors, Numerical Methods in Laminar and Turbulent Flow. Plymouth, Pentech Press, 1978.

8. B. Ramaswamy, Finite Element Solution for Advection and Natural Convection Flows, Comp. Fluids, vol. 16, pp. 349-388, 1988.

9. R. Rannacher, On Chorin Projection Method for the Incompressible Navier-Stokes Equations, Lecture Notes in Mathematics, vol. 1530, pp. 167-183, 1993.

10. O.C. Zienkiewicz, and R. Codina, A General Algorithm for Compressible and Incompressible Flow, Part I. The split Characteristic Based Scheme, Int. J. Numer. Meth. Fluids, vol, 20, pp. 869-85, 1995. 
11. O.C. Zienkiewicz, P. Nithiarasu, R. Codina, M. Vázque, and P. Ortiz, The Characteristic Based Split Procedure: an Efficient and Accurate Algorithm for Fluid Problems, Int. J. Numer. Meth. Fluids, vol. 31, pp. 359-392, 1999.

12. P. Nithiarasu, R. Codina, and O.C. Zienkiewicz, The Characteristic-Based Split (CBS) Scheme-a Unified Approach to Fluid Dynamics, Int. J. Numer. Meth. Engng, vol. 66, pp. 1514-1546, 2006.

13. P. Nithiarasu, A Unified Fractional Step Method for Compressible and Incompressible Flows, Heat Transfer and Incompressible Solid Mechanics, vol. 18, pp. 111-130, 2008.

14. A. Shamekhi, and K. Sadeghy, On the Use of Characteristic-Based Split Mesh-free Method for Solving Flow Problems, Int. J. Numer. Meth. Fluids, vol. 56, pp. 18851907, 2008.

15. M. Nickaeen, and A. Ashrafizadeh, A semi-implicit CBS Finite Volume Algorithm for the Solution of Incompressible Flow Problems, Proc. $18^{\text {th }}$ annual conference, vol. 1 , CFD society of Canada, London, Canada, 2010.

16. M. Nickaeen, and A. Ashrafizadeh, A Characteristic-Based Split Finite Volume Algorithm for the Solution of Incompressible Flow Problems, Proc. Fifth European Conference on Computational Fluid Dynamics, vol. 1, pp. 177, ECCOMAS CFD, Lisbon, Portugal, 2010.

17. M. Nickaeen, and A. Ashrafizadeh, Comparison between two Characteristic-Based Split Finite Volume Methods for the Solution of Incompressible Flow Problems, Proc. $13^{\text {th }}$ Annual \& $2^{\text {nd }}$ International Fluid Dynamics Conference, vol. 1, pp. 33, FD2010, Shiraz, Iran, 2010.

18. RW. Lewis, P. Nithiarasu, and KN. Seetharamu, Fundamentals of the Finite Element Method for Heat and Fluid Flow, $1^{\text {st }}$ ed., Wiley, NJ, 2004.

19. U. Ghia, K.N. Ghia, and C.T. Shin, High-resolution for Incompressible Flow using the Navier-Stokes Equations and Multigrid Method, J. Comp. Phys., vol. 48, pp. 387411, 1982.

20. B. F. Armaly, F. Durst, J. C. F. Pereira, and B. Schoenung. Experimental and Theoretical Investigation of Backward-Facing Step Flow, J. Fluid Mech., vol. 127, pp. 473-496, 1983. 
21. D. de Vahl Davis, Natural Convection of Air in a Square Cavity: A Bench Mark Solution, Int. J. Numer. Meth. Fluids, vol. 3, pp. 249-264, 1983.

22. DC. Wan, BSV. Patnaik, and GW. Wei, A New Benchmark Quality Solution for the Buoyancy-driven Cavity by Discrete Singular Convolution, Numerical Heat Transfer: Part B, vol. 40, pp. 199-228, 2001. 
Table 1. Comparison of maximum (Max) and minimum (Min) velocities and their corresponding positions along the mid lines in lid-driven cavity flow

\begin{tabular}{lllccccc}
\hline$R e$ & Reference & Mesh & $u_{\text {Min }}$ & $y_{\text {Min }}$ & $v_{\text {Max }}$ & $v_{\text {Min }}$ & $x_{\text {Min }}$ \\
\hline \multirow{4}{*}{400} & CBS & 1 & -0.2971 & 0.268 & 0.2758 & -0.4187 & 0.863 \\
& CBS & 2 & -0.3341 & 0.283 & 0.3079 & -0.4597 & 0.864 \\
& Ghia [19] & $129 \times 129$ & -0.3258 & 0.282 & 0.3059 & -0.4537 & 0.856 \\
\hline \multirow{4}{*}{1000} & CBS & 1 & -0.3371 & 0.161 & 0.3241 & -0.4682 & 0.911 \\
& CBS & 2 & -0.3885 & 0.174 & 0.3770 & -0.5261 & 0.911 \\
& Ghia [19] & $129 \times 129$ & -0.3829 & 0.172 & 0.3709 & -0.5155 & 0.906 \\
\hline \multirow{4}{*}{5000} & CBS & 1 & -0.3869 & 0.065 & 0.3838 & -0.4962 & 0.958 \\
& CBS & 2 & -0.4303 & 0.074 & 0.4315 & -0.5358 & 0.957 \\
& Ghia [19] & $129 \times 129$ & -0.4321 & 0.068 & 0.4387 & -0.5504 & 0.951 \\
\hline
\end{tabular}


Table 2. Comparison of fully explicit and semi-implicit schemes in BFS flow at different $R e$

\begin{tabular}{|c|c|c|c|}
\hline$R e$ & & fully explicit & semi-implicit \\
\hline 100 & Iteration & 57,079 & 13,663 \\
\hline $\mathrm{X}_{1} /(\mathrm{H}-\mathrm{h})_{[20]}=2.83$ & $\begin{array}{l}\text { Time (s) } \\
\mathrm{X}_{1} /(\mathrm{H}-\mathrm{h})\end{array}$ & $\begin{array}{c}1,286 \\
2.86\end{array}$ & $\begin{array}{l}528 \\
2.88\end{array}$ \\
\hline 300 & Iteration & 77,890 & 25,976 \\
\hline $\mathrm{X}_{1} /(\mathrm{H}-\mathrm{h})_{[20]}=6.54$ & $\begin{array}{l}\text { Time (s) } \\
\mathrm{X}_{1} /(\mathrm{H}-\mathrm{h})\end{array}$ & $\begin{array}{c}11,051 \\
6.72\end{array}$ & $\begin{array}{c}5,662 \\
6.76\end{array}$ \\
\hline 400 & Iteration & 87,105 & 27,333 \\
\hline $\mathrm{X}_{1} /(\mathrm{H}-\mathrm{h})_{[20]}=8.46$ & $\begin{array}{l}\text { Time (s) } \\
\mathrm{X}_{1} /(\mathrm{H}-\mathrm{h})\end{array}$ & $\begin{array}{c}12,450 \\
8.28\end{array}$ & $\begin{array}{c}5982 \\
8.44\end{array}$ \\
\hline
\end{tabular}

Table 3. Grid studies, maximum values of vertical and horizontal velocities along the mid-width and mid-height lines at $R a=10^{3}$

\begin{tabular}{lcccc}
\hline \multirow{2}{*}{ Grid } & \multicolumn{2}{c}{$\max (u)$} & \multicolumn{2}{c}{$\max (v)$} \\
& fully explicit & semi-implicit & fully explicit & semi-implicit \\
\hline $21 \times 21$ & 3.6482 & 3.6351 & 3.5493 & 3.5048 \\
$41 \times 41$ & 3.6488 & 3.6448 & 3.6583 & 3.6499 \\
$81 \times 81$ & 3.6491 & 3.6484 & 3.6874 & 3.6860 \\
$161 \times 161$ & 3.6492 & 3.6490 & 3.6947 & 3.6892 \\
\hline
\end{tabular}


Table 4. Comparison of buoyancy-driven flow quantities and their corresponding positions in parentheses with the benchmark solution, $10^{3}<R a<10^{6}$

\begin{tabular}{|c|c|c|c|c|}
\hline$R a$ & Quantity & Ref. [22] & Ref. [21] & Present \\
\hline \multirow{4}{*}{$10^{3}$} & Max. (u) & $\begin{array}{l}3.489 \\
(0.813)\end{array}$ & $\begin{array}{l}3.65 \\
(0.813)\end{array}$ & $\begin{array}{l}3.6491 \\
(0.8161)\end{array}$ \\
\hline & Max. (v) & $\begin{array}{l}3.686 \\
(0.188)\end{array}$ & $\begin{array}{l}3.70 \\
(0.178)\end{array}$ & $\begin{array}{l}3.6874 \\
(0.1761)\end{array}$ \\
\hline & Max. $(N u)$ & $\begin{array}{l}1.501 \\
(0.08)\end{array}$ & $\begin{array}{l}1.50 \\
(0.092)\end{array}$ & $\begin{array}{l}1.5053 \\
(0.0865)\end{array}$ \\
\hline & Min. $(N u)$ & $\begin{array}{l}0.691 \\
(1.0)\end{array}$ & $\begin{array}{l}0.692 \\
(1.0)\end{array}$ & $\begin{array}{l}0.6904 \\
(1.0)\end{array}$ \\
\hline \multirow{4}{*}{$10^{4}$} & Max. (u) & $\begin{array}{l}16.122 \\
(0.815)\end{array}$ & $\begin{array}{l}16.2 \\
(0.823)\end{array}$ & $\begin{array}{l}16.112 \\
(0.8283)\end{array}$ \\
\hline & Max. (v) & $\begin{array}{l}19.79 \\
(0.12)\end{array}$ & $\begin{array}{l}19.62 \\
(0.119)\end{array}$ & $\begin{array}{l}19.5249 \\
(0.1145)\end{array}$ \\
\hline & Max. $(N u)$ & $\begin{array}{l}3.597 \\
(0.13)\end{array}$ & $\begin{array}{l}3.53 \\
(0.143)\end{array}$ & $\begin{array}{l}3.5276 \\
(0.1481)\end{array}$ \\
\hline & Min. $(N u)$ & $\begin{array}{l}0.577 \\
(1.0)\end{array}$ & $\begin{array}{l}0.586 \\
(1.0)\end{array}$ & $\begin{array}{l}0.5775 \\
(1.0)\end{array}$ \\
\hline \multirow{4}{*}{$10^{5}$} & Max. (u) & $\begin{array}{l}33.39 \\
(0.835)\end{array}$ & $\begin{array}{l}34.73 \\
(0.855)\end{array}$ & $\begin{array}{l}33.583 \\
(0.8519)\end{array}$ \\
\hline & Max. $(v)$ & $\begin{array}{l}70.63 \\
(0.072)\end{array}$ & $\begin{array}{l}68.6 \\
(0.066)\end{array}$ & $\begin{array}{l}67.2172 \\
(0.0639)\end{array}$ \\
\hline & Max. $(N u)$ & $\begin{array}{l}7.945 \\
(0.08)\end{array}$ & $\begin{array}{l}7.71 \\
(0.08)\end{array}$ & $\begin{array}{l}7.6563 \\
(0.0834)\end{array}$ \\
\hline & Min. $(N u)$ & $\begin{array}{l}0.698 \\
(1.0)\end{array}$ & $\begin{array}{l}0.729 \\
(1.0)\end{array}$ & $\begin{array}{l}0.6725 \\
(1.0)\end{array}$ \\
\hline \multirow{4}{*}{$10^{6}$} & Max. (u) & $\begin{array}{l}65.40 \\
(0.86)\end{array}$ & $\begin{array}{l}64.3 \\
(0.850)\end{array}$ & $\begin{array}{l}60.954 \\
(0.8479)\end{array}$ \\
\hline & Max. (v) & $\begin{array}{l}227.11 \\
(0.040)\end{array}$ & $\begin{array}{l}219.36 \\
(0.038)\end{array}$ & $\begin{array}{l}213.289 \\
(0.0391)\end{array}$ \\
\hline & Max. $(N u)$ & $\begin{array}{l}17.86 \\
(0.03)\end{array}$ & $\begin{array}{l}17.92 \\
(0.038)\end{array}$ & $\begin{array}{l}17.2705 \\
(0.039)\end{array}$ \\
\hline & Min. $(N u)$ & $\begin{array}{l}0.9132 \\
(1.0)\end{array}$ & $\begin{array}{l}0.989 \\
(1.0)\end{array}$ & $\begin{array}{l}0.8634 \\
(1.0)\end{array}$ \\
\hline
\end{tabular}


Figure 1. A cell-centered finite volume grid.

Figure 2. (a) Lid-driven cavity flow, geometry and boundary conditions, (b) Mesh 1, $85 \times 85$, (c) Mesh 2, $129 \times 129$

Figure 3. Lid-driven cavity flow, comparison with Ghia et al. [19] for the $v$-velocity profile along the horizontal center line. (a) $R e=400$, (b) $R e=1000$.

Figure 4. Lid-driven cavity flow, comparison with Ghia et al. [19] for the $u$-velocity profile along the vertical center line. (a) $R e=400$, (b) $R e=1000$.

Figure 5. Lid-driven cavity flow at $R e=5000$ on mesh 2, comparison with Ghia et al. [19] of velocity profiles along the center lines. (a) $u$ velocity profile, (b) $v$ velocity profile.

Figure 6. BFS flow, geometry and boundary conditions.

Figure 7. Sample finite volume grid in BFS flow, step region grid distribution.

Figure 8. BFS flow, comparison of reattachment and separation lengths with experimental results [20].

Figure 9. Buoyancy-driven cavity flow, geometry and boundary conditions.

Figure 10. Natural-convection patterns simulated by FV CBS, $10^{3}<R a<10^{6}(a)$ streamlines, $(b)$ iso- $u$ contours, $(c)$ iso- $v$ contours, $(d)$ isotherms. 


\begin{tabular}{|c|c|c|c|c|}
\hline - & • & $\bullet$ & • & - \\
\hline$\bullet$ & $\begin{array}{l}\mathrm{NW} \\
\bullet\end{array}$ & $\stackrel{N}{\bullet}$ & NE & - \\
\hline$\bullet$ & $\mathrm{W} \bullet$ & $\mathrm{P}$ & - $\mathrm{E}$ & • \\
\hline • & $\stackrel{\bullet}{\mathrm{sW}}$ & $\stackrel{\bullet}{S}$ & $\stackrel{\bullet}{\mathrm{SE}}$ & $\bullet$ \\
\hline$\bullet$ & • & $\bullet$ & - & • \\
\hline
\end{tabular}

Figure 1 


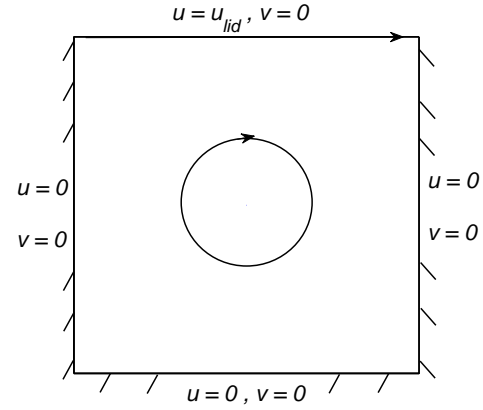

(a)

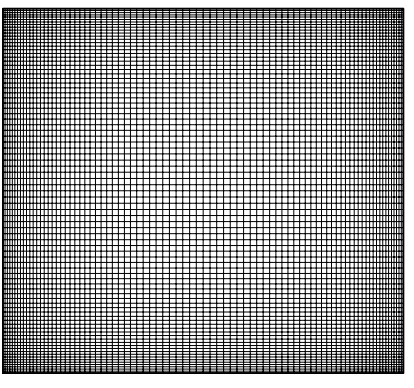

(b)

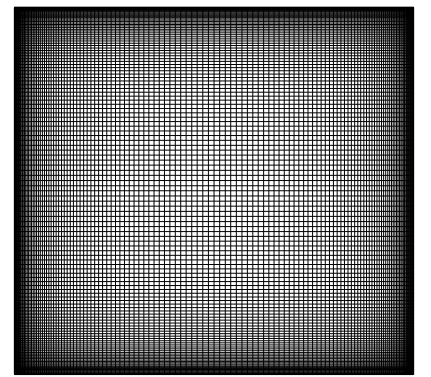

(c)

Figure 2 


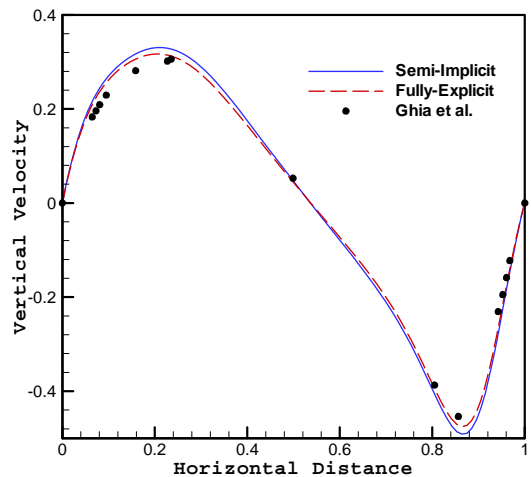

(a)

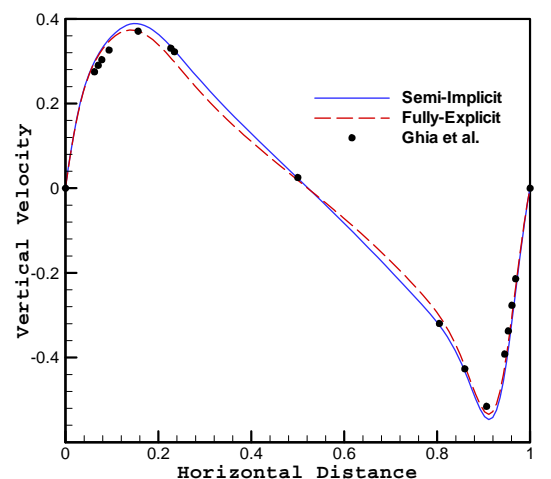

(b)

Figure 3 


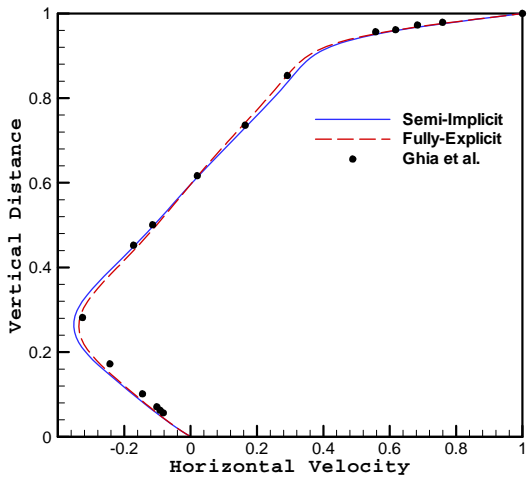

(a)

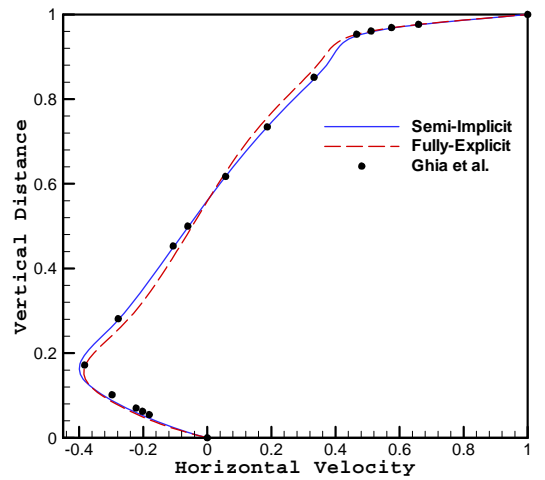

(b)

Figure 4 


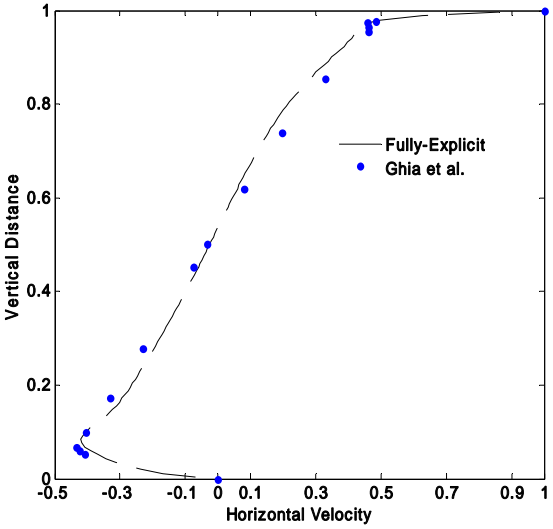

(a)

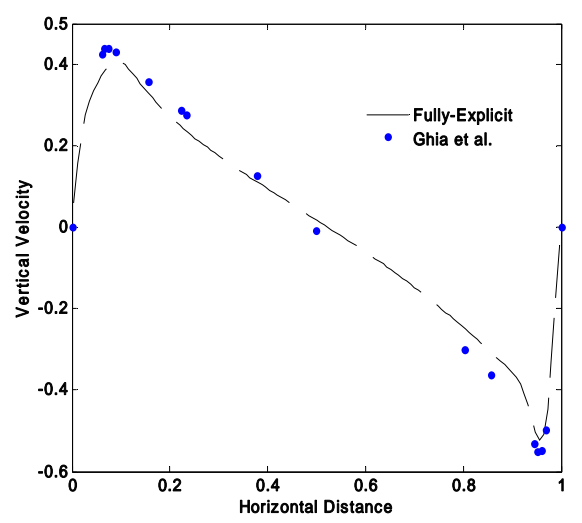

(b)

Figure 5 


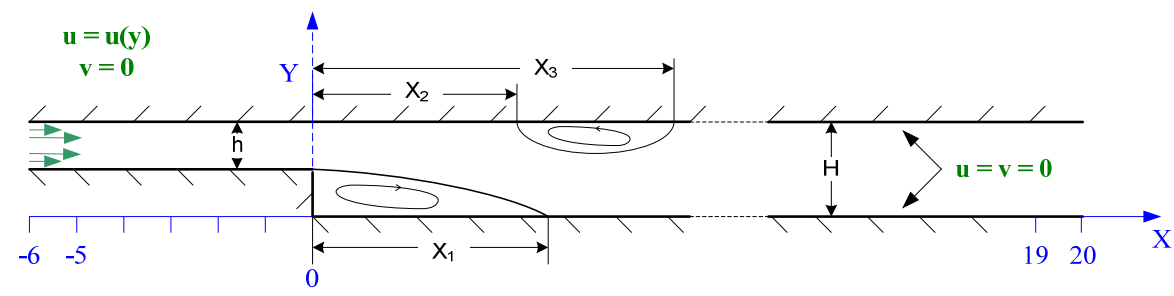

Figure 6 


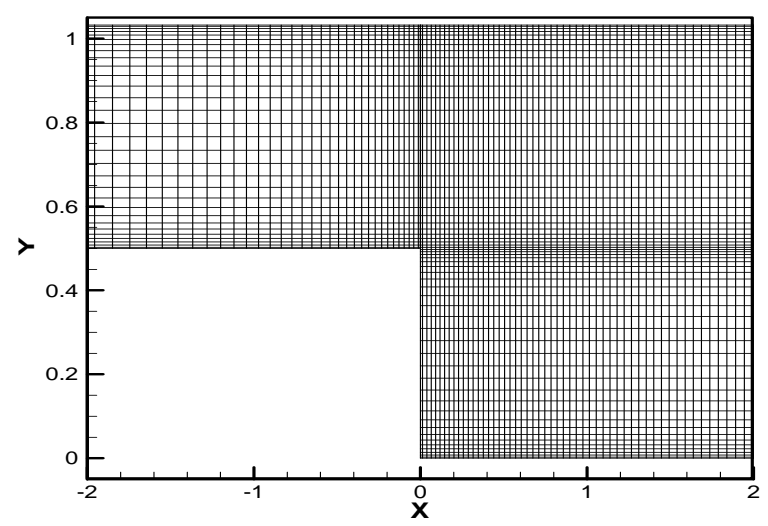

Figure 7 


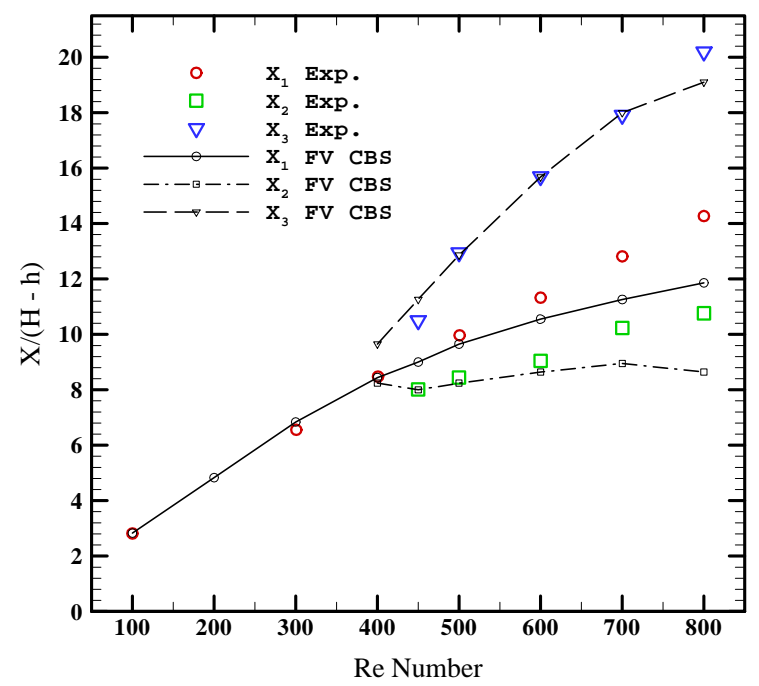

Figure 8 


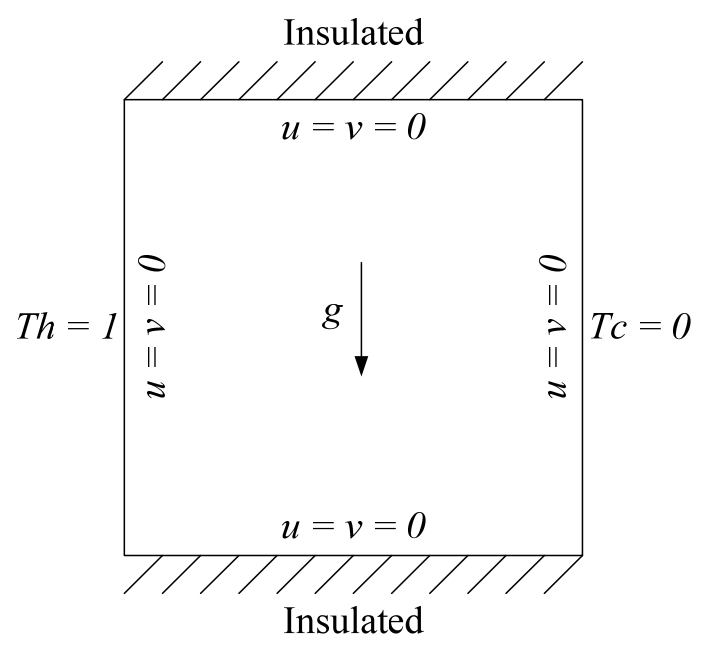

Figure 9 

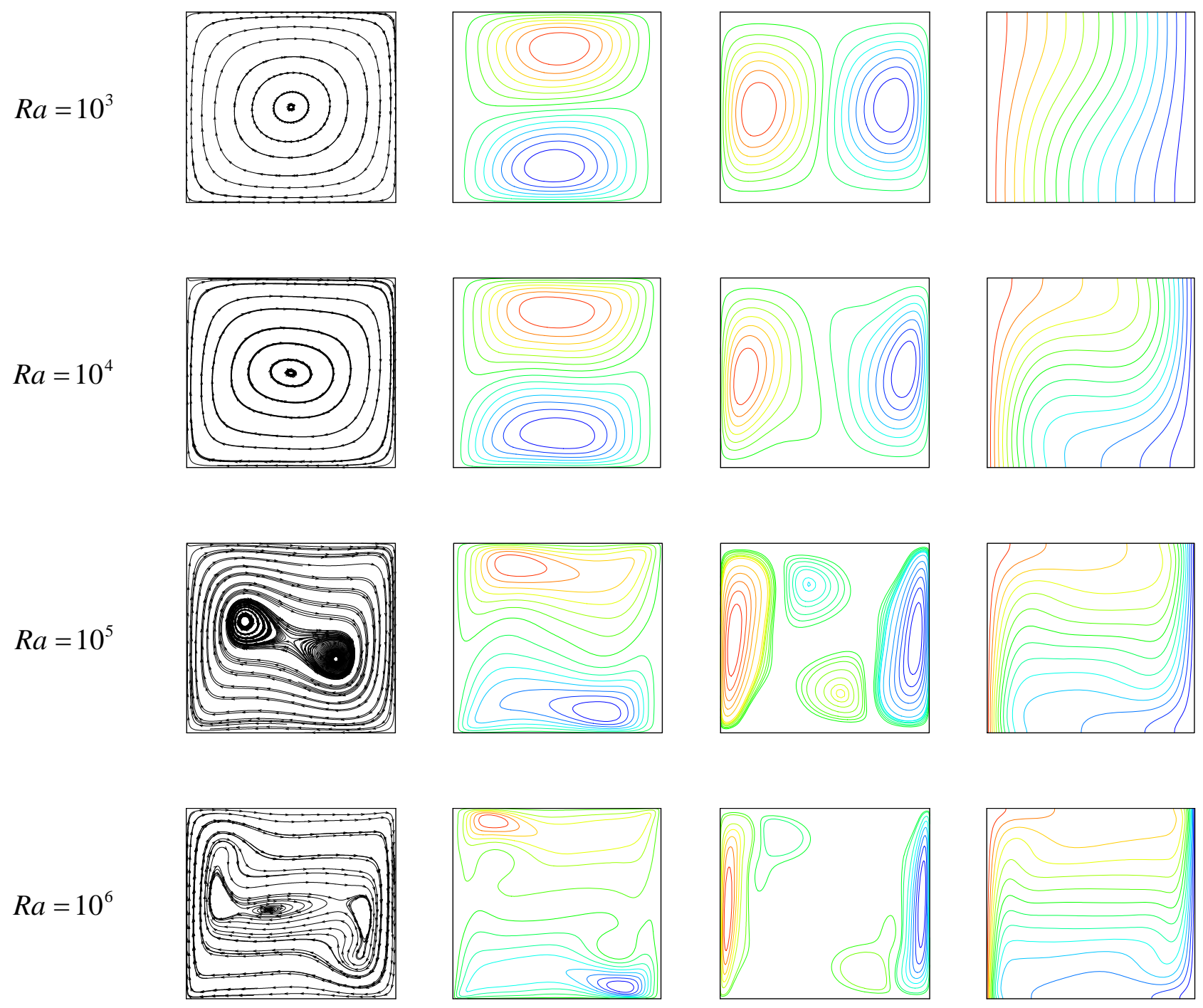

(a)

(b)

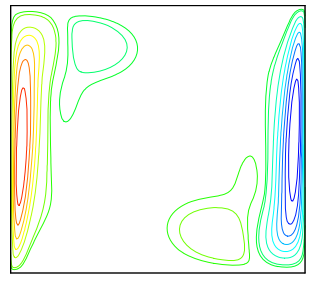

(c)

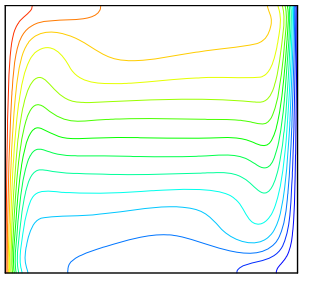

(d)

Figure 10 